\title{
Practical Methods for Measuring the Tortuosity of Porous Materials from Binary or Gray-Tone Tomographic Reconstructions
}

\author{
Cedric J. Gommes
}

Dept. of Chemical Engineering, University of Liège B6A, Allée du 6 août 3, B-4000 Liège, Belgium

\section{Anton-Jan Bons}

ExxonMobil Chemical Europe Inc., European Technology Center, Hermeslaan 2, B-1831 Machelen, Belgium

\section{Silvia Blacher}

Dept. of Chemical Engineering, University of Liège B6A, Allée du 6 août 3, B-4000 Liège, Belgium

John H. Dunsmuir and Andy H. Tsou

ExxonMobil Research and Engineering Co., Corporate Strategic Research, 1545 Route 22 East, Annandale, NJ 08801-3059

DOI 10.1002/aic.11812

Published online June 23, 2009 in Wiley InterScience (www.interscience.wiley.com).

Two practical methods are proposed to measure the tortuosity of a porous or permeable material from its tomographic reconstruction. The first method is based on the direct measurement of the shortest distance between two points in the pores, and the second is based on the geodesic reconstruction of the pore or permeation space. Unlike the first method, the second can be directly applied to gray-tone tomograms, without the need of a segmentation step. The methods are illustrated with an electron tomogram of clay/plastic nanocomposite, an X-ray microtomogram of sandstone, and a series of model morphologies consisting of penetrable random spheres. For the latter series, the measured tortuosities compare very well with those derived independently from the theoretical effective diffusion coefficients. () 2009 American Institute of Chemical Engineers AIChE J, 55: 2000-2012, 2009

Keywords: tortuosity, porous materials, tomography, image analysis, transport phenomena

\section{Introduction}

A host of imaging techniques are nowadays available commercially to investigate the three-dimensional structure of materials, ranging from the nanometer scale with electron tomography, ${ }^{1}$ focused ion beam tomography, ${ }^{2}$ to the micro-

\footnotetext{
Correspondence concerning this article should be addressed to C. J. Gommes at cedric.gommes@ulg.ac.be.

(C) 2009 American Institute of Chemical Engineers
}

meter scale with X-ray microtomography ${ }^{3}$ and confocal microscopy, ${ }^{4}$ just to mention the more widely known techniques. ${ }^{5}$ These recent technological progress offer the prospect of advancing our understanding and control of materials performance under working conditions, by starting with an accurate knowledge of their microstructure.

The various imaging techniques provide the users with three-dimensional gray-tone images, but to make such a dataset useful, the complex information contained in the 3D images must be condensed into a few easy-to-handle 
morphological parameters. The purpose of this article is to present different methods that can be used to estimate the tortuosity of porous materials from their tomographic reconstruction. In this context, we use the term "porous material" not only for the conventional concept of a material containing open space or voids, but also for a compound material containing phases with different permeability; the phase with the highest permeability is then referred to as the pore space.

In porous materials, tortuosity characterizes the sinuosity and interconnectedness of pore space. It is a concept common to many models that aim at relating macroscopic transport properties of porous materials to their microstructure. ${ }^{6}$ It is used for phenomena as diverse as molecular diffusion, fluid permeation, electrical or thermal conduction, and sound propagation. ${ }^{7}$ In practice, tortuosity is often an empirical factor that is used to match a posteriori experimental data to a specific model. When tortuosity is used for making predictions, crude approximations are generally used. For instance, in the context of molecular diffusion it is common practice to equate the tortuosity to the inverse of the porosity. ${ }^{8}$ Therefore, although the qualitative meaning of tortuosity is clear, it is a loosely defined quantity. The exact geometrical meaning of tortuosity in the context of various transport phenomena may be differently dependent, say, on pore size or the presence of bottlenecks. This article focuses on the geometrical tortuosity, which is defined as the ratio of the length of the shortest path connecting two points in the pore space to the straight line distance between them.

In a first section of the article, three-dimensional images are presented on the basis of which the methods of the article are discussed. The first image is an electron tomogram of a clay/plastic nanocomposite and the second image is an $\mathrm{X}$-ray tomogram of sandstone. A series of 3D images of model porous materials with known effective diffusion coefficients is also presented. In a second section, two different algorithms are proposed and tested to measure the tortuosity. They are based on numerical tools that are available in the general purpose engineering software package Matlab ${ }^{\circledR}$. Finally, in the discussion section, the relation between the two measures of tortuosity is derived; it is shown that tortuosity can be defined in several different ways, all mathematically sound.

The methods proposed in this article can be used to measure a tortuosity parameter from the observed microstructure, which can then be correlated with macroscopically measured transport phenomena on one hand, and, e.g., the fabrication methods on the other hand. As such, these methods can contribute to a better fundamental understanding of structure-property relationships, which is key to the evaluation or development of materials.

\section{Materials and Methods \\ Clay/plastic nanocomposite}

The first example in this study is a clay/plastic nanocomposite which has potential applications in packaging. Exfoliated clay lamellae of a few nanometers thick and tens to hundreds of nanometers wide are dispersed in a plastic matrix (in this case ethylene vinyl acetate or EVA) to reduce gas diffusion through the compound. Electron tomography in
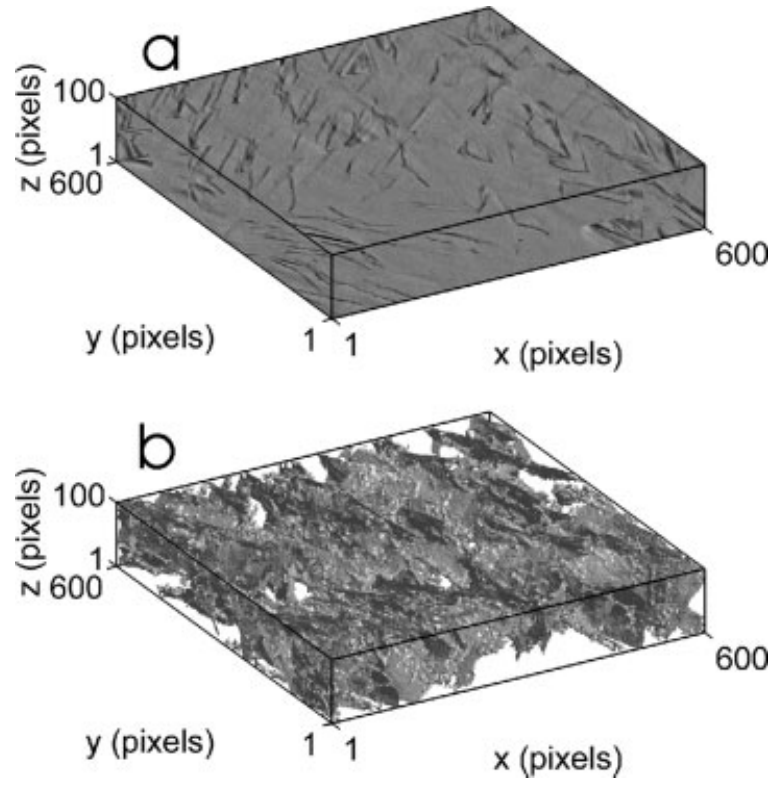

Figure 1. Electron tomography reconstruction of a clay/plastic nanocomposite: (a) original graytone tomogram (dark = clay, gray = plastic) and (b) segmented impermeable clay lamellae.

The physical size of a pixel is $1.6 \mathrm{~nm}$.

STEM-HAADF mode ${ }^{9}$ was used to image the 3D microstructure at the nanometer level; Figure 1a shows the original gray-tone tomogram of this clay-plastic nanocomposite.

The gray-tone tomogram was segmented to discriminate between the pixels of the plastic and of the clay phases. This was done in two steps: (i) First, all pixels with intensity lower than a given threshold (chosen manually) are said to belong to the clay lamellae. (ii) After the thresholding, morphological closing was applied ${ }^{10,11}$ to remove many spurious holes a few pixels across in the lamellae in the segmented tomogram. The obtained segmented impermeable clay lamellae tomogram is represented in Figure 1b.

\section{Sandstone}

The second example is a porous sandstone (Figure 2a). Sandstone is a typical reservoir rock for fluids such as water, oil and gas, and its porosity and permeability are important factors controlling the amount of fluid in the reservoir, and the rate at which it can migrate or at which it can be extracted. This sample consists largely of quartz $\left(\mathrm{SiO}_{2}\right)$ grains (gray in Figure 2a), with minor quantities of other minerals like clay or calcium carbonate (white in Figure 2a). $\mathrm{X}$-ray micro-tomography ${ }^{12,13}$ was used to analyze the $3 \mathrm{D}$ microstructure; the 3D reconstruction is shown in Figure 2a.

The gray-tone tomogram was segmented using the same general methodology as in "Clay/plastic nanocomposite" section. The obtained binary $3 \mathrm{D}$ image is represented in Figure $2 \mathrm{~b}$.

\section{Fully penetrable sphere model}

The last 3D images we shall consider is a series of model morphologies made up of fully penetrable monodispersed 

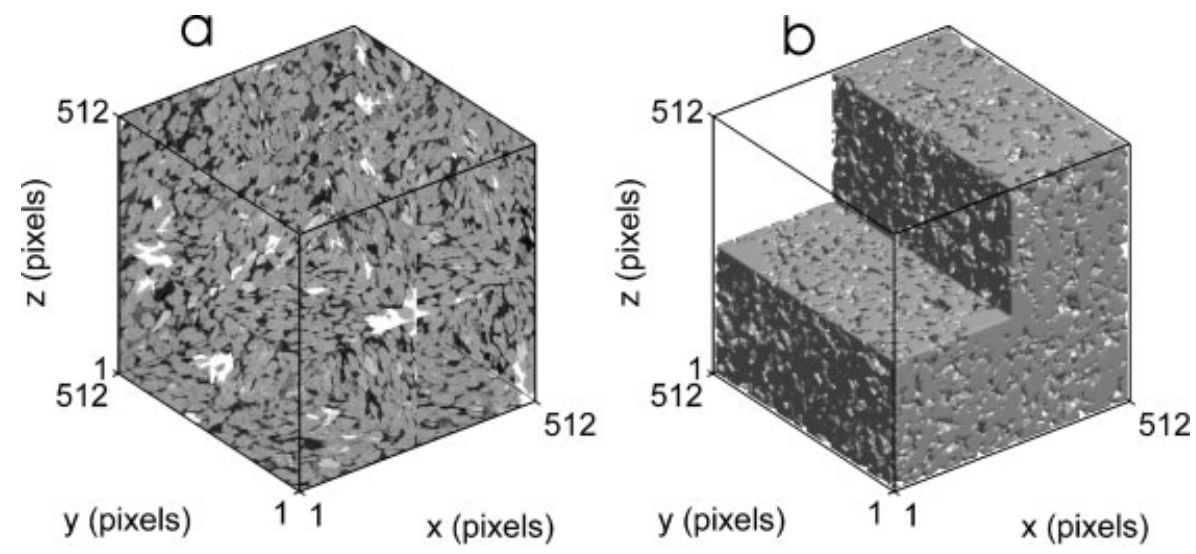

Figure 2. X-ray microtomography reconstruction of a sandstone: (a) original gray-tone tomogram (dark = pores, gray = quartz, bright $=$ carbonate cement) and (b) segmented tomogram.

The physical size of a pixel is $5.72 \mu \mathrm{m}$.

spheres, distributed in space according to a 3D Poisson process. ${ }^{13,14}$ We shall refer to it hereafter as the fully penetrable sphere model (FPS); the overlapping spheres are considered to be the solid phase of the porous material. Figure 3 shows realizations of the model with nominal porosities of $0.2,0.5$, and 0.8 . The porosity is calculated theoretically as

$$
\phi=\exp \left(-\rho \frac{4}{3} \pi R^{3}\right)
$$

where $R$ is the radius of each individual sphere, and $\rho$ is density of the associated Poisson point process, i.e., the average number of spheres per unit volume. ${ }^{13,14}$ The word nominal highlights the fact that the actual porosity of a single realization may differ slightly from the theoretical average value.

Nine realizations of the FPS model were generated on a $3 \mathrm{D}$ grid of size $250 \times 250 \times 250$ pixels, with the radius of each sphere set to $R=7.5$ pixels, and $\rho$ chosen according to Eq. 1 to yield nominal porosities from $\phi=0.1$ to 0.9 , with a step of 0.1 . For a porosity larger than about $\phi=0.7$, the spheres do not all touch each other (see Figure $3 \mathrm{c}$ ); this is not problematic for the purpose of the present article.

Theoretical calculations of the effective diffusivity of a molecule within the pore space of the FPS model have been done by Kim and Torquato, ${ }^{15}$ which enables to assess the relevance of proposed methodology to transport phenomena. The effective diffusivity $D_{\mathrm{e}}$ of a molecule in a porous solid can be expressed as ${ }^{6-8}$

$$
\frac{D_{\mathrm{e}}}{D_{\mathrm{m}}}=\frac{\phi}{\tau^{2}}
$$

where $D_{\mathrm{m}}$ is the molecular diffusivity unhindered by any pore wall, $\phi$ is the porosity, and $\tau$ is the tortuosity of the pore space. Inverting Eq. 2, the effective diffusivity can be converted into a tortuosity: the tortuosity derived from the values of $D_{\mathrm{e}} / D_{\mathrm{m}}$ published by Kim and Torquato is plotted as a solid line in Figure 4. In the literature, the tortuosity is sometimes introduced in Eq. 2 with an exponent $1^{8}$; it has to be noted, however, that in this case, it is merely used as a tortuosity factor with no precise geometrical interpretation. Arguments are given in Appendix A, according to which it is the square of the tortuosity that should enter Eq. 2, if $\tau$ is to be given a geometrical meaning.

\section{Results}

\section{Tortuosity measurement based on distances}

Given two points in the pore space, the geometrical tortuosity is defined as the ratio of $L_{\mathrm{G}}$, the length of the shortest
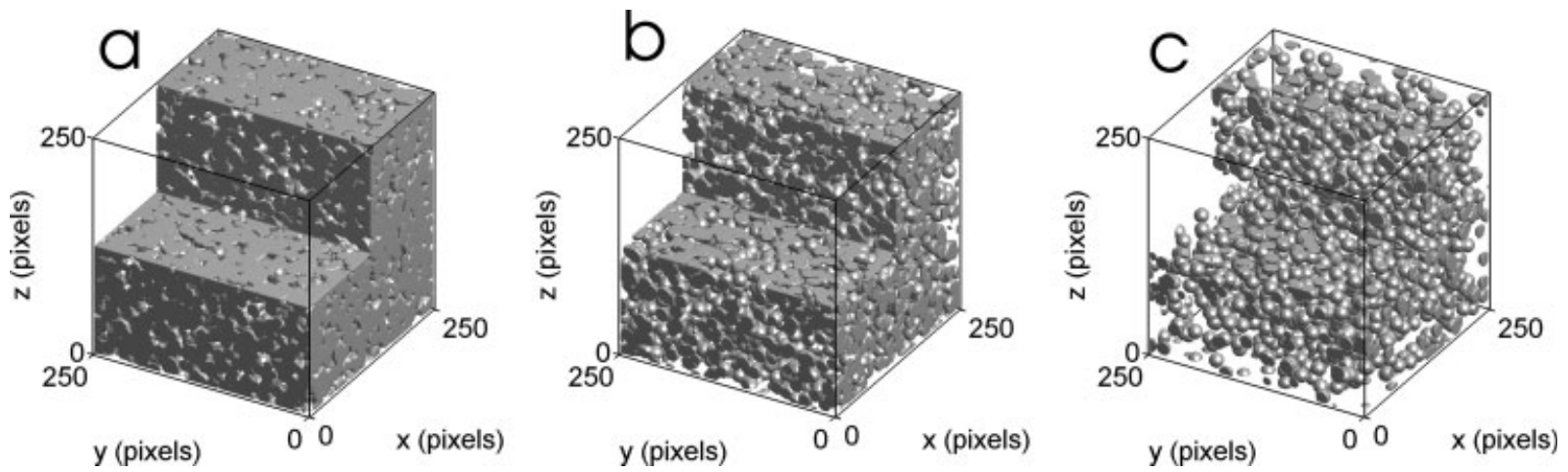

Figure 3. Realizations of a Poisson sphere model with nominal porosity 0.2 (a), 0.5 (b), and 0.8 (c). 


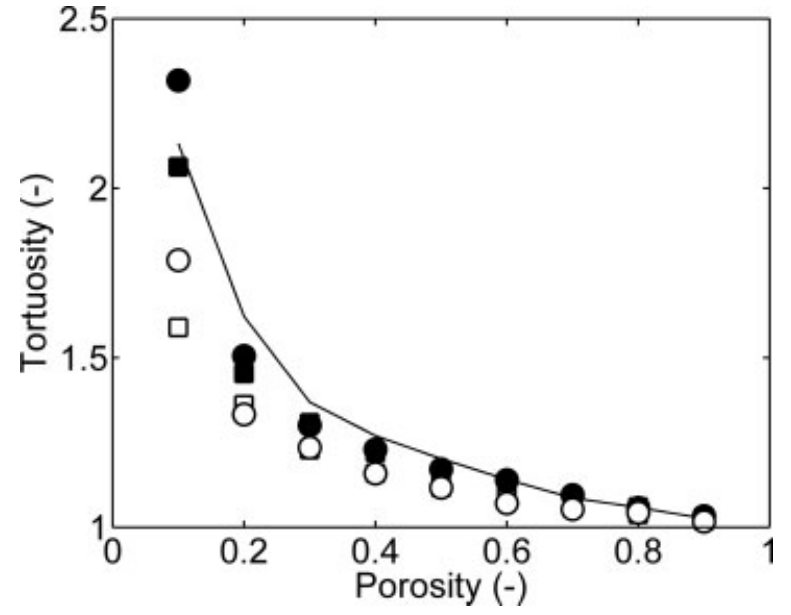

Figure 4. Relation between tortuosity and porosity of the Poisson spheres model.

The solid line is the theoretical tortuosity obtained from the effective diffusivity calculated by Kim and Torquato. ${ }^{14}$ The symbols are estimations based on geodesic distance (circles) and on geodesic reconstruction (square); full and empty symbols are from the initial and final slopes, respectively.

path that connects them and lies entirely within the pore space, to $L_{\mathrm{E}}$, the straight line distance between them. ${ }^{7}$ The subscripts $\mathrm{E}$ and $\mathrm{G}$ stand for Euclidean and geodesic, which are the names that these two distances receive in the frame of morphological image analysis. ${ }^{10,13}$ A direct way of measuring $\tau$ would be to choose couples of points in the pore space of a binary image, and to calculate the geodesic and Euclidean distances between them. If the distance between the two points is too short, say, only a few times the average pore size, the ratio $L_{\mathrm{G}} / L_{\mathrm{E}}$ may not be relevant to the effective transport properties of the porous material. A more useful and precise definition of the tortuosity would therefore be

$$
\tau=\lim _{L_{\mathrm{G}} \rightarrow \infty} \frac{L_{\mathrm{G}}}{L_{\mathrm{E}}}
$$

For large values of $L_{\mathrm{G}}$, it can be noted that the locus of all points at a given geodesic (or Euclidean) distance from another point is practically a plane surface. Therefore, the tortuosity can be conveniently measured by comparing the geodesic and Euclidean distances between planes rather than points, as illustrated in Figure 5.

Figure 5 shows the geodesic distance from the $y=0$ plane of part of the sandstone to any point of its pore space, using a 6-connectivity of the pixels. Every pixel in the pore space is characterized by its Euclidean and geodesic distances to the $y=0$ plane; let $\rho\left(L_{\mathrm{E}}, L_{\mathrm{G}}\right)$ be the joint distribution of $L_{\mathrm{E}}$ and $L_{\mathrm{G}}$, such that $\rho d L_{\mathrm{E}} d L_{\mathrm{G}}$ be proportional to the number of points with Euclidean distance in $\left[L_{\mathrm{E}}, L_{\mathrm{E}}+d L_{\mathrm{E}}\right]$ and geodesic distance in $\left[L_{\mathrm{G}}, L_{\mathrm{G}}+d L_{\mathrm{G}}\right]$. The joint distribution corresponding to Figure 5 is shown in Figure 6. The distribution is roughly centered on an oblique line, which points to the fact that $L_{\mathrm{E}}$ and $L_{\mathrm{G}}$ are linearly correlated. The average value of $L_{\mathrm{G}}$ corresponding to a given $L_{\mathrm{E}}$ is referred to hereafter as $\left\langle L_{\mathrm{G}}\right\rangle$. It is defined as

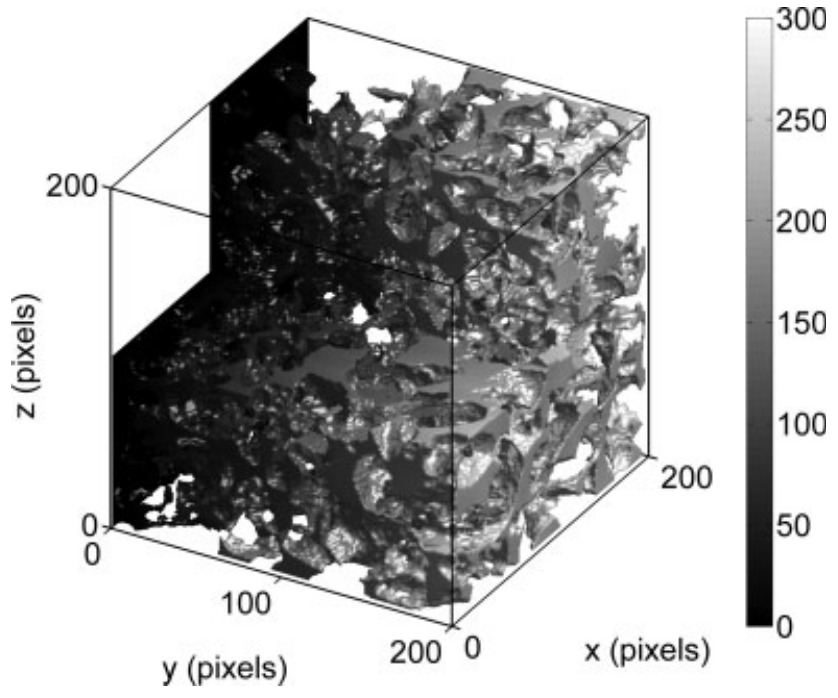

Figure 5. Geodesic distance, within the pore space of the sandstone, from the $y=0$ limit of the image to any pixel in the pores: the gray tone of the image corresponds to the shortest distant within the pore space between any given point and the $y=0$ plane.

Note that the $y$ coordinate is the Euclidean distance in the same direction.

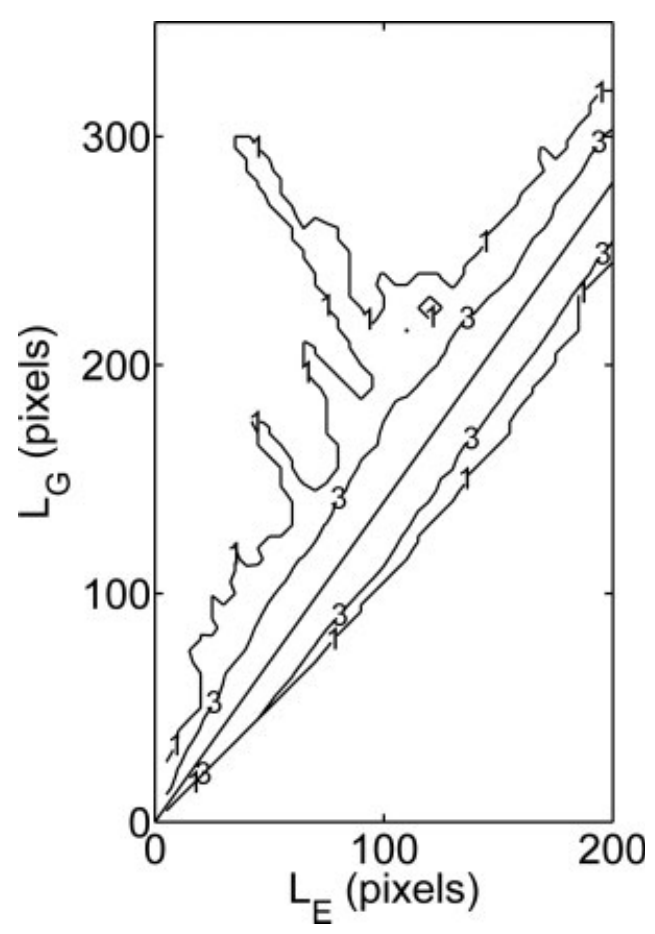

Figure 6. Isolines of the joint distribution $\rho\left(L_{E}, L_{G}\right)$ of Euclidean $\left(L_{E}\right)$ and of geodesic $\left(L_{G}\right)$ distances corresponding to Figure 5: the values shown are proportional to the decimal logarithm of the number of pixels with Euclidean distance in $\left[L_{E}, L_{E}+d L_{E}\right]$ and geodesic distance in $\left[L_{G}, L_{G}+d L_{G}\right]$. 

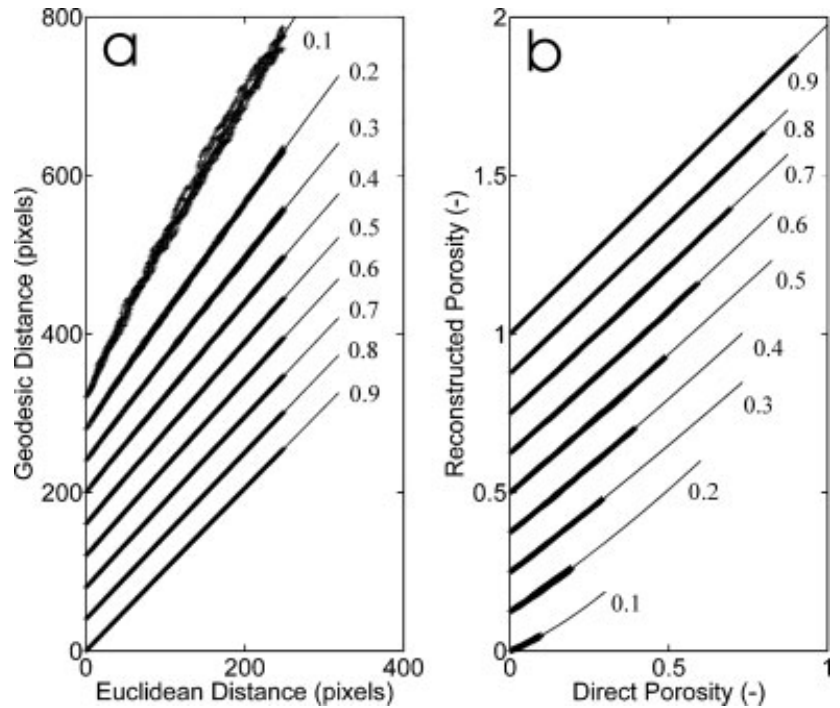

Figure 7. Assessment of the tortuosity of Poisson spheres models with various porosities (ranging from 0.1 to 0.9 ): (a) Relation between geodesic and Euclidean distance, (b) relation between reconstructed porosity and direct porosity.

For each realization, six independent measurements are plotted, corresponding to measurements starting from the six faces of the same cubic tomogram. The solid lines are parabolic fits and the curves are shifted vertically.

$$
\left\langle L_{\mathrm{G}}\right\rangle=\int_{0}^{\infty} \rho\left(L_{\mathrm{E}}, L_{\mathrm{G}}\right) L_{\mathrm{G}} d L_{\mathrm{G}} / \int_{0}^{\infty} \rho\left(L_{\mathrm{E}}, L_{\mathrm{G}}\right) d L_{\mathrm{G}}
$$

The average geodesic distance $\left\langle L_{\mathrm{G}}\right\rangle$ is plotted as a function of $L_{\mathrm{E}}$ in Figure 7a, 8a and 9a, for the FPS model, for the sandstone, and for the clay/plastic composite, respectively.

In the case of the FPS model, the points obtained with the distances calculated from any of the six external faces of the tomograms overlap almost perfectly (Figure $7 \mathrm{a}$ ). The relation between $\left\langle L_{\mathrm{G}}\right\rangle$ and $L_{\mathrm{E}}$ is slightly curvilinear. The $\left\langle L_{\mathrm{G}}\right\rangle$ vs. $L_{\mathrm{E}}$ curves are fitted with a parabola; the initial and final slopes of which are reported in Figure 4 as full and empty symbols, respectively.

The tortuosity of the sandstone was measured by cutting the original tomogram of size $512^{3}$ pixels into eight images of size $256^{3}$ pixels. In each image, the geodesic distance was calculated starting from each of the six possible faces, which gives a total of 16 estimations in each direction of space. Each of the three series plotted in Figure 8a is a superposition of 16 independent measurements. A slight downward deviation in the curves is also observed. The initial slopes of $\left\langle L_{\mathrm{G}}\right\rangle$ vs. $L_{\mathrm{E}}$ were estimated via a parabolic fit; they are reported in Table 1 as $\tau$. A similar procedure was used for the clay/plastic nanocomposite (see Figure 9a); the corresponding values are also reported in Table 1.

Note that for the sandstone, $\tau$ is the same in all directions indicating an isotropic porosity. On the other hand, the clay/
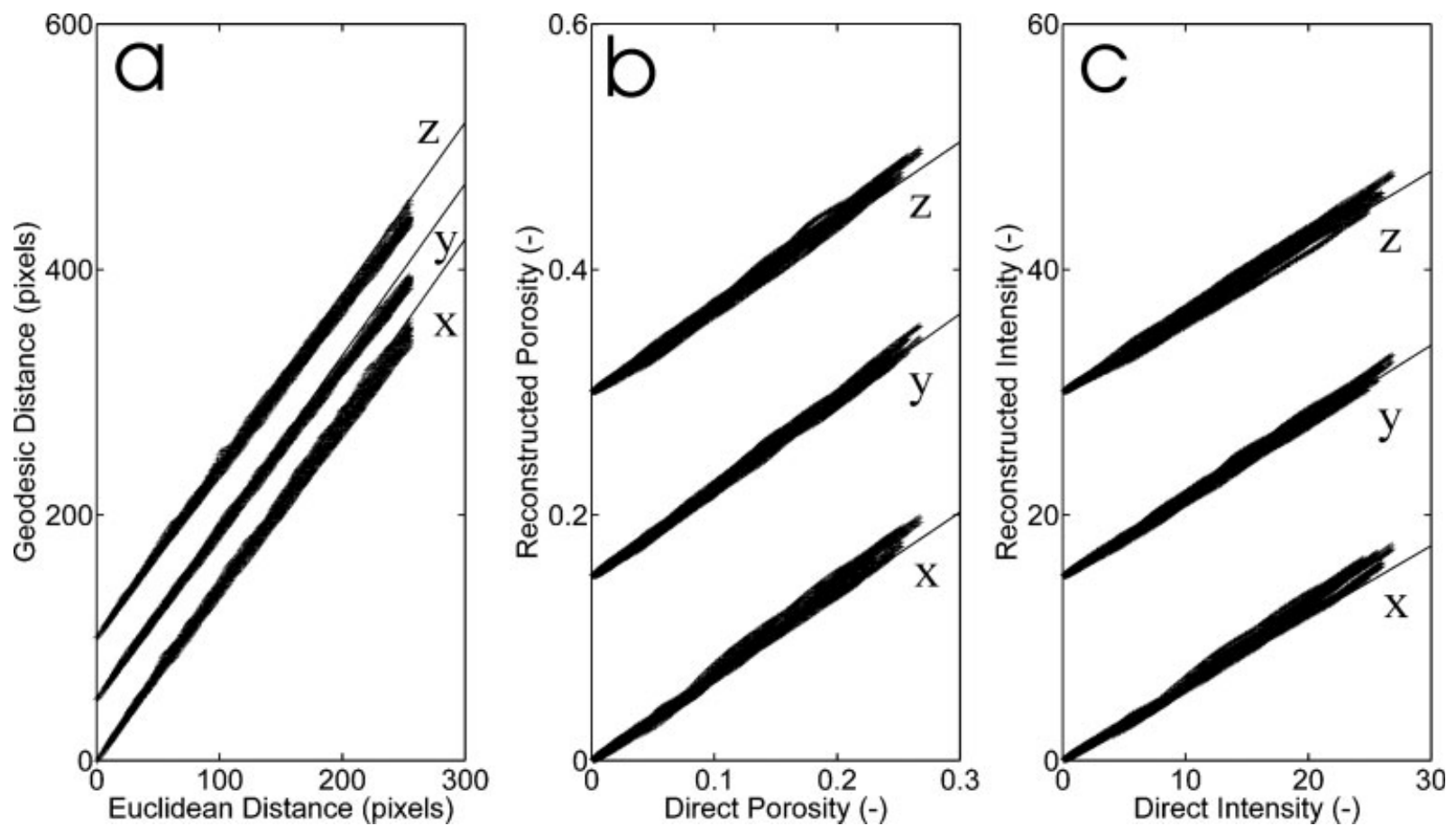

Figure 8. Three different measures of the tortuosity of the sandstone, based on (a) the geodesic distance within the pore space of the segmented image (Figure 4), (b) the geodesic reconstruction of pore space of the binary image (Figure 10, first row), and (c) the geodesic reconstruction of the gray-tone image (Figure 10, second row).

The three series in each graph correspond to the three orthogonal directions of Figure 2; the curves are arbitrarily shifted vertically. 
Table 1. Different Estimates of the Tortuosity of the Sandstone and of the Clay/Plastic Composite, in the Three Orthogonal Directions of Figures 3 and 4

\begin{tabular}{|c|c|c|c|c|c|c|}
\hline & \multicolumn{3}{|c|}{ Sandstone } & \multicolumn{3}{|c|}{ Clay/Plastic Composite } \\
\hline & $x$ & $y$ & $z$ & $x$ & $y$ & $z$ \\
\hline$\tau$ & $1.42 \pm 0.01$ & $1.40 \pm 0.01$ & $1.40 \pm 0.02$ & $1.19 \pm 0.02$ & $1.06 \pm 0.01$ & $1.09 \pm 0.01$ \\
\hline$\tau_{\mathrm{r}}$ & $1.48 \pm 0.04$ & $1.40 \pm 0.03$ & $1.47 \pm 0.04$ & $1.20 \pm 0.02$ & $1.05 \pm 0.01$ & $1.10 \pm 0.01$ \\
\hline$\tau_{\mathrm{r}}^{\prime}$ & $1.71 \pm 0.05$ & $1.59 \pm 0.04$ & $1.67 \pm 0.06$ & $1.06 \pm 0.003$ & $1.03 \pm 0.001$ & $1.04 \pm 0.002$ \\
\hline
\end{tabular}

The values are expressed as mean \pm standard error of the mean. $\tau$, tortuosity based on distances; $\tau_{\mathrm{r}}$, tortuosity based on binary reconstruction; $\tau_{\mathrm{r}}^{\prime}$, tortuosity based on gray-tone reconstruction.

plastic nanocomposite is anisotropic, with $\tau$ ca. $10 \%$ higher in the $x$-direction as compared to the $y$ - and $z$-directions.

\section{Tortuosity measurement based on geodesic reconstruction}

The method presented in "Tortuosity measurement based on distances" section can only be used on segmented tomograms, on which geodesic distances can be defined. The starting point of the measurements has to be a binary image as in Figures $1 b$ and $2 b$, which requires preliminary processing steps. The segmentation step can be very dependent on the user's subjectivity, in the choice of an intensity threshold, in the criteria that are used to remove spurious objects, etc. In the present section, we propose a method to measure the tortuosity on the gray-tone tomograms themselves (i.e., directly from Figures $1 \mathrm{a}$ and $2 \mathrm{a}$ ) without the need of any preprocessing step. We will first describe the principle of geodesic reconstruction for binary images, and then expand it to gray-tone images.

Let us consider the geodesic reconstruction of the pore space, starting from one limit of the tomogram. The notion of geodesic reconstruction is summarized in appendix B, and it is illustrated in Figure 10 on the same tomogram as used in Figure 5. Because the pores are tortuous, their geodesic reconstruction progresses through the tomogram slower than what may be called a direct reconstruction (the limit of which is shown by a transparent plane in Figure 10). The more tortuous the pore space, the slower the reconstruction.

A quantitative link with the tortuosity can be made on the basis of the single narrow and tortuous pore sketched in Figure 11. The relation between the geodesic distance $L_{\mathrm{G}}$ along the pore and the Euclidean distance $L_{\mathrm{E}}$ along the same pore is

$$
L_{\mathrm{G}}=\int_{0}^{L_{\mathrm{E}}} \frac{d l_{\mathrm{E}}}{\cos (\theta)}
$$

where $\theta$ is the local angle between the direction of the pore and the direction along which the Euclidean distance is measured. From Eq. 3, one has

$$
\tau=\lim _{L_{\mathrm{E}} \rightarrow \infty} \frac{1}{L_{\mathrm{E}}} \int_{0}^{L_{\mathrm{E}}} \frac{d l_{\mathrm{E}}}{\cos (\theta)}=\left\langle\frac{1}{\cos (\theta)}\right\rangle
$$

where the brackets denote the average value. Therefore, the tortuosity can be thought of as the average of the reciprocal director cosine of the pores.
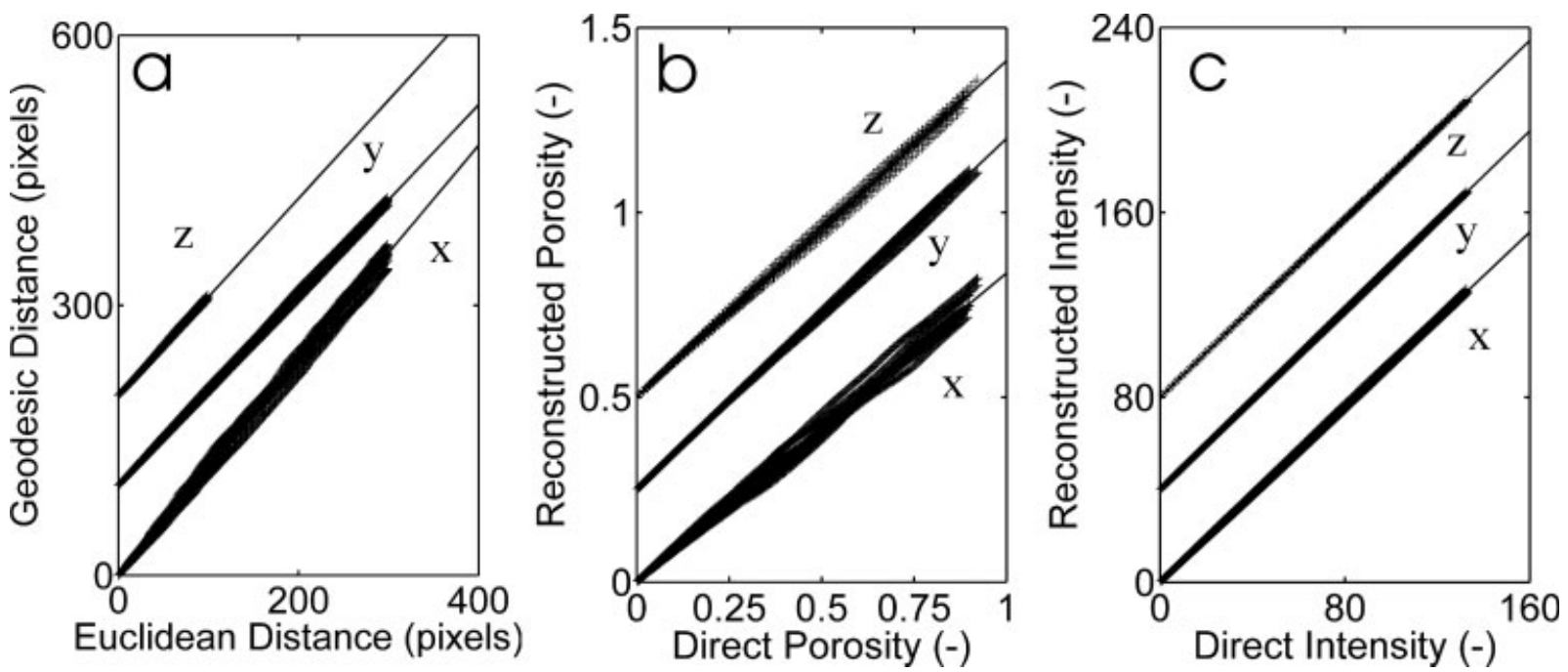

Figure 9. Three different measures of the tortuosity of the clay/plastic composite based on (a) the geodesic distance within the plastic phase of the segmented image (Figure 4), (b) the geodesic reconstruction of plastic phase of the binary image (Figure 10, first row), and (c) the geodesic reconstruction of the graytone image (Figure 10, second row).

The three series in each graph correspond to the three orthogonal directions of Figure 1; the curves are arbitrarily shifted vertically. 

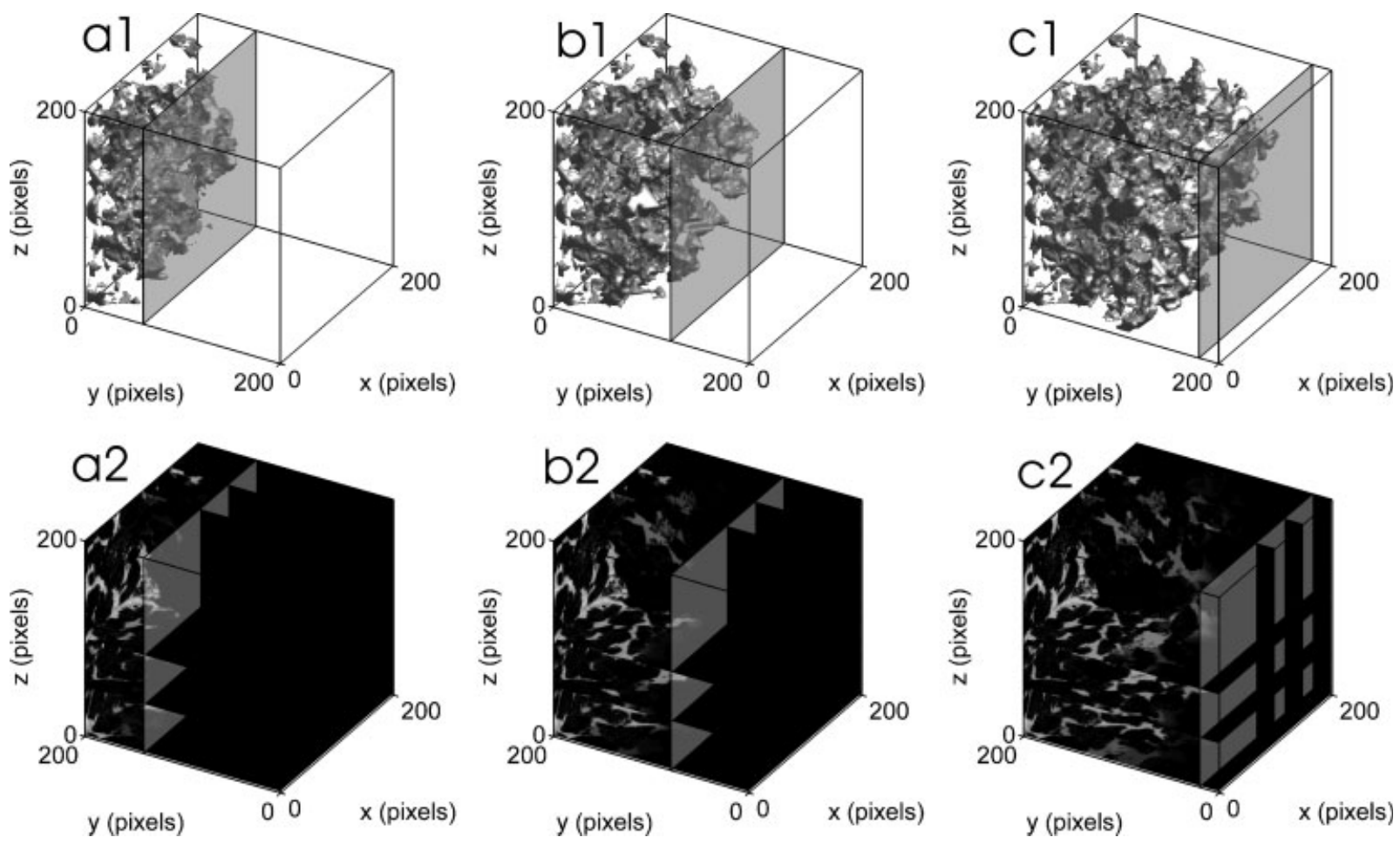

Figure 10. Geodesic reconstruction of the pore space of the sandstone starting from the $y=0$ limit of the image: binary reconstruction (top row) and gray-tone reconstruction (bottom row).

The $y$ coordinate of the transparent plane is the number of reconstruction steps at each stage; the plane would be the limit of the reconstructed volume if the tortuosity were equal to 1 .

A binary reconstruction is done by adding repeatedly a 1-pixel-thick layer to the reconstructed pore space, as illustrated in Figure 11a for a geodesic reconstruction, and in Figure $11 \mathrm{~b}$ for a so-called direct reconstruction. Because the geodesic reconstruction always progresses in the local direction of the pore, the volume of the reconstructed pore (expressed in pixels) after $n$ geodesic dilations is

$$
V_{\mathrm{G}}(n)=S_{\perp} \times n
$$

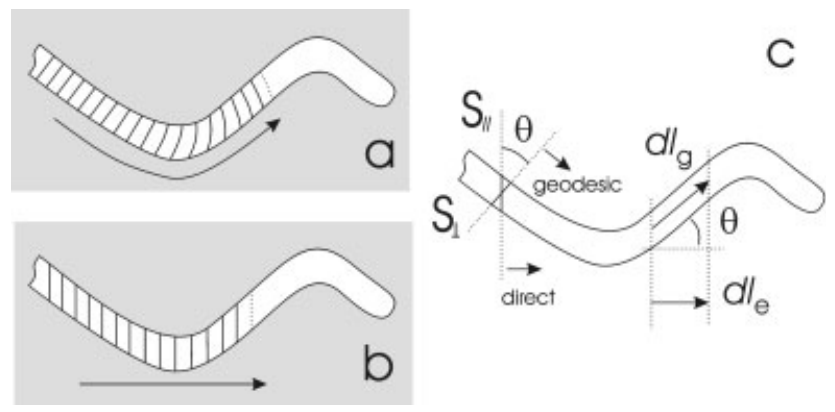

Figure 11. Sketches (a) of the geodesic reconstruction and (b) of the direct reconstruction of a narrow tortuous pore.

Useful symbols are defined in (c), notably the local director angle $\theta$, and the areas $S_{\perp}$ and $S_{/}$of the cross sections of the pore in the directions perpendicular to the geodesic and direct reconstructions, respectively. where $S_{\perp}$ is the cross section area, that is constant all along the pore. A similar reasoning applies to the direct reconstruction (Figure 11b) but the relevant area is $S_{/ /}$, parallel to the starting plane of reconstruction. As $S_{/ /}$depends on the local orientation of the pore, one has

$$
V_{\mathrm{D}}(n)=\left\langle S_{/ /}\right\rangle \times n
$$

where $\left\langle\mathrm{S}_{/ /}\right\rangle$is the value of $S_{/ /}$averaged all along the pore. From Figure 11c one finds easily that $\left\langle\mathrm{S}_{/ /}\right\rangle=S_{\perp}\langle 1 / \cos (\theta)\rangle$. Combined with Eq. 6, the latter result shows that the slope of the $V_{\mathrm{D}}(n)$ vs. $V_{\mathrm{G}}(n)$ curve can also serve as a measure of the tortuosity; we shall refer to it as $\tau_{\mathrm{r}}$

$$
\tau_{\mathrm{r}}=\frac{V_{\mathrm{D}}(n)}{V_{\mathrm{G}}(n)}
$$

where the subscript $r$ stands for reconstruction. The reasoning leading to Eq. 9 is based on the simplified situation of Figure 11; a more general relation between $\tau_{\mathrm{r}}$ and $\tau$, valid for any pore structure, is derived in "Nonuniqueness of the tortuosity" section.

Figures $7 \mathrm{~b}, 8 \mathrm{~b}$, and $9 \mathrm{~b}$ plot the $V_{\mathrm{G}}$ vs. $V_{\mathrm{D}}$ curves for the FPS models, for the sandstone, and for the clay/plastic composite, respectively. For the geodesic reconstruction, a 6-connectivity of the pixels was assumed. The reconstructed and direct volumes are both normalized to the total volume of the image, so that they correspond to porosities. In 
particular, the largest normalized value of $V_{\mathrm{D}}$ in the figures is the actual porosity of the solids. In the same way as for $L_{\mathrm{G}}$ vs. $L_{\mathrm{E}}$, a slight deviation from linearity is observed. The tortuosities of the FPS models, determined from the initial and final slopes, are plotted in Figure 4 as full and empty symbols. For the sandstone and for the clay/plastic composite, only the initial slope is reported in Table 1 as $\tau_{\mathrm{r}}$. Again, it is evident that the clay/plastic nanocomposite is anisotropic.

The tortuosity based on the geodesic reconstruction can be measured on gray-tone tomograms directly, without having to segment the tomograms into solid space and pore space. The principle of gray-tone geodesic reconstruction is described in appendix B, and it is illustrated in Figures $10 \mathrm{a} 2-\mathrm{c} 2$. This figure is based on the negative of the tomogram, such that the pore space is bright and the solid phase is dark. The volume of a gray-tone image is defined as the sum of the intensity of all its pixels; we shall use the notations $\bar{V}_{\mathrm{D}}(n)$ and $\bar{V}_{\mathrm{G}}(n)$. By analogy with Eq. 9, the gray-tone tortuosity $\tau_{\mathrm{r}}^{\prime}$ is defined as

$$
\tau_{\mathrm{r}}^{\prime}=\frac{\bar{V}_{\mathrm{D}}(n)}{\bar{V}_{\mathrm{G}}(n)}
$$

Figures $8 \mathrm{c}$ and $9 \mathrm{c}$ are the $\bar{V}_{\mathrm{D}}(n)$ vs. $\bar{G}_{\mathrm{D}}(n)$ plots resulting from the gray-tone reconstructions of the sandstone and of the clay/ plastic composite. In the case of the sandstone, the intensity of the tomograms was inverted first, as in Figure 10, to make the pore space bright (with high intensities) and the solid phase dark (with low intensities). For the clay/plastic tomogram, the contrast was already set such that the bright phase represents the more permeable plastic phase, the tortuosity of which is to be determined. The corresponding average values of $\tau_{\mathrm{r}}^{\prime}$ are reported in Table 1 together with the standard error of the mean. The anisotropy of the clay/plastic nanocomposite is less pronounced in this case.

\section{Discussion}

\section{Finite size effects}

The origin of the downward bend in the curves of $\left\langle L_{\mathrm{G}}\right\rangle$ vs. $L_{\mathrm{E}}$ (notably in Figures $7 \mathrm{a}$ and $8 \mathrm{a}$ ) can be found in the finite size of the tomograms. For instance, in the case of the sandstone in Figure 5 not all pore points at Euclidean distance $y=200$ pixels can be reached from a pore path that starts at $y=0$ pixels. The pore paths that connect the $y=0$ plane to these points are so tortuous that they exit the range of the tomogram. Therefore, the points that would most contribute to increase $\left\langle L_{\mathrm{G}}\right\rangle$ are simply ignored, which leads to a negative bias in the estimation of the tortuosity.

This is seen even more clearly in the joint distribution $\rho\left(L_{\mathrm{E}}, L_{\mathrm{G}}\right)$ of the Euclidean and geodesic distances (Figure 6). For low $L_{\mathrm{E}}, \rho$ exhibits features in which $L_{\mathrm{G}}$ is negatively correlated with $L_{\mathrm{E}}$; these features correspond to dead-end pores that point towards $y=0$. They contribute to increase $\left\langle L_{\mathrm{G}}\right\rangle$ at low $L_{\mathrm{E}}$, but they are spuriously absent for large values of $L_{\mathrm{E}}$ because the distant dead-end pores that point toward $y=0$ cannot be reached by a path that lies entirely within the tomogram. The distant dead-ends are therefore ignored in the statistics.
The bend in the $V_{\mathrm{R}}$ vs. $V_{\mathrm{D}}$ curves, seen in Figures $7 \mathrm{~b}$ and $8 \mathrm{~b}$, has the same origin. It leads to an underestimation of the tortuosity over large distances. For that reason, the tortuosity was estimated from the initial slopes of the curves, via a parabolic fit. The difference between the empty and full symbols in Figure 4 is therefore a finite size effect.

\section{Nonuniqueness of the tortuosity}

Heuristic arguments were given in "Tortuosity measurement based on geodesic reconstruction" section for the equality of $\tau$ and $\tau_{\mathrm{r}}$ in the case of a simple pore structure made up of a collection of non connected narrow tortuous pores. Any real porous structure is more complex, with interconnected pores of various sizes. In the present section, we first derive a general relation between $\tau$ and $\tau_{\mathrm{r}}$, based on the joint probability distribution of $L_{\mathrm{E}}$ and $L_{\mathrm{G}}$.

All the morphological information relevant to the tortuosity is contained in the joint distribution $\rho\left(L_{\mathrm{E}}, L_{\mathrm{G}}\right)$ of the Euclidean and geodesic distances, an example of which is given in Figure 6. The possible functional form or $\rho\left(L_{\mathrm{E}}, L_{\mathrm{G}}\right)$ is constrained by two general considerations about the homogeneity of the material. First, in the case where $L_{\mathrm{E}}$ is a distance from a plane (as e.g. in Figure 5) homogeneity requires that the total number of pore pixels per unit area $S$ of the starting plane be equal to the porosity $\phi$, independently of $L_{\mathrm{E}}$. This implies

$$
\int_{0}^{\infty} \rho\left(L_{\mathrm{G}}, L_{\mathrm{E}}\right) d L_{\mathrm{G}}=S \phi
$$

Second, the assumption of large scale homogeneity of the material is tantamount to assuming that-for values of $L_{\mathrm{E}}$ much larger than the pore size, the sphere diameter in the FPS model, etc.- the pore space possesses no characteristic length scale. Therefore, dimensional arguments demand that the dependence of $\rho$ on $L_{\mathrm{E}}$ and $L_{\mathrm{G}}$ be only through their ratio $L_{\mathrm{G}} / L_{\mathrm{E}}$. Together with Eq. 11, the latter argument implies that the only possible functional form of $\rho$ is

$$
\rho\left(L_{\mathrm{G}}, L_{\mathrm{E}}\right)=\frac{S \phi}{L_{\mathrm{E}}} f\left(\frac{L_{\mathrm{G}}}{L_{\mathrm{E}}}\right)
$$

where $f$ is a function characteristic of the pore structure, satisfying $\int_{0}^{\infty} f(x) d x=1$. As the geodesic distance cannot be smaller than the Euclidean distance, $f$ also satisfies $f(x)=0$ for $x<1$.

From Eqs. 4 and 12, the average geodesic distance $\left\langle L_{\mathrm{G}}\right\rangle$ is found to be equal to

$$
\left\langle L_{\mathrm{G}}\right\rangle=L_{\mathrm{E}} \int_{1}^{\infty} x f(x) d x
$$

which enables to identify the tortusoity $\tau$ with the first moment of $f$. As for the reconstruction tortuosity $\tau_{\mathrm{r}}$, one has to note first that the pore volume resulting from $n$ direct reconstruction steps is 


$$
V_{\mathrm{D}}(n)=\int_{0}^{n} d L_{\mathrm{E}} \int_{L_{\mathrm{E}}}^{\infty} d L_{\mathrm{G}} \rho\left(L_{\mathrm{G}}, L_{\mathrm{E}}\right)
$$

which, from Eq. 11, simply implies $V_{\mathrm{D}}(n)=\phi S n$. To relate the volume of the geodesic reconstruction $V_{\mathrm{G}}(n)$ to $\rho\left(L_{\mathrm{E}}, L_{\mathrm{G}}\right)$, let us remark that the geodesic distance at a given point is equal to the number of geodesic dilations needed for the reconstructed set to reach that point. Put mathematically, this means

$$
V_{\mathrm{G}}(n)=\int_{0}^{n} d L_{\mathrm{G}} \int_{0}^{\infty} d L_{\mathrm{E}} \rho\left(L_{\mathrm{G}}, L_{\mathrm{E}}\right)
$$

Combining Eqs. 12, 14, and 15 leads to

$$
\frac{V_{\mathrm{G}}(n)}{V_{\mathrm{D}}(n)}=\int_{1}^{\infty} \frac{1}{x} f(x) d x
$$

which, combined with Eq. 9, enables us to identify $1 / \tau_{\mathrm{r}}$ with the moment of order -1 of $f$.

In the case of a porous material characterized by a very peaked function $f$, the two measures of the tortuosity $\tau$ and $\tau_{\mathrm{r}}$ are very similar. The narrow and independent tortuous pores of Figure 11 are characterized by $f(x)=\delta(x-\tau)$, where $\delta$ is Dirac's function. In that particular case $\tau=\tau_{\mathrm{r}}$, in agreement with the heuristic arguments of "Tortusity measurement based on geodesic reconstruction" section. In general, however, the two estimates have no reason to coincide. In the case of the sandstone, $\tau_{\mathrm{r}}$ is slightly larger than $\tau$ (Table 1), while in the case of the FPS model (Figure 4) the opposite is observed.

\section{Gray-tone tortuosity}

We now analyze how the contrast and the background level of the gray-tone tomograms influence the estimated value of the reconstruction tortuosity $\tau_{\mathrm{r}}{ }^{\prime}$. Let us first recall that it is the tortuosity of the brightest phase that is estimated, so that the solid phase has the lowest intensity $I_{0}$, and the pore space has a higher intensity, say $I_{0}+\Delta I$ where $\Delta I$ is a positive contrast. The intensity $I(x)$ of the gray-tone image at any spatial position $x$ is modeled as

$$
I(x)=I_{0}+\Delta I \Phi(x)
$$

where $\Phi(x)$ is the indicator function of the pores [e.g., Ref. 14], which takes the value 1 in the pores, and 0 in the solid phase. The volume of a gray-tone image is the sum of the intensity of all its pixels. In the case of a direct reconstruction of an image with intensity given by Eq. 17, the reconstructed volume after $n$ direct steps is

$$
\bar{V}_{\mathrm{D}}(n)=\operatorname{Sn}\left[I_{0}+\Delta I \phi\right]
$$

where $\phi$ is the porosity. Equation 18 reduces to the binary expression derived from Eq. 14 for $I_{0}=0$ and $\Delta I=1$, as it should.
For estimating the volume of the gray-tone geodesic reconstruction after $n$ steps, it has to be noted that the reconstruction of the background intensity is not hindered by the presence of tortuous pores, because the latter have a larger intensity. Accordingly, one has

$$
\bar{V}_{\mathrm{G}}(n)=S n I_{0}+\Delta I V_{\mathrm{G}}(n)
$$

Combining Eqs. 9, 10, 18, and 19 leads to the following relation between the gray-tone tortuosity $\tau_{\mathrm{r}}^{\prime}$ and the binary tortuosity $\tau_{\mathrm{r}}$

$$
\frac{\tau_{\mathrm{r}}^{\prime}}{\tau_{\mathrm{r}}}=\frac{1+\phi \Delta I / I_{0}}{\tau_{\mathrm{r}}+\phi \Delta I / I_{0}}
$$

A general conclusion that can be drawn from Eq. 20 is

$$
1 \leq \tau_{\mathrm{r}}^{\prime} \leq \tau_{\mathrm{r}}
$$

which means that, provided the gray-tone image intensity obeys Eq. 17, the gray-tone tortuosity should underestimate the actual tortuosity. In Eq. 21, the lower limit is attained for small values of $\phi \Delta I / I_{0}$, i.e., for poorly contrasted tomograms; the upper limit is attained for large values of $\phi \Delta I / I_{0}$, i.e., for low values of the background intensity $I_{0}$. The present analysis helps understanding the values of $\tau_{\mathrm{r}}$ found for the clay/plastic composite (Table 1). The low contrast, in agreement with Eq. 20 , leads to severe underestimation of $\tau_{\mathrm{r}}$ that also contributes to mask the anisotropy of the material. Another conclusion drawn from Eq. 20 is that the detrimental effect of a low contrast becomes worse if the material has a low porosity.

For the sandstone, the fact that the gray-tone tortuosity is larger than the binary tortuosity points to a fact that has not been taken into account for deriving Eq. 20. Any real image is characterized by a point spread function, so that the transition of tomogram intensity between the pore and solid is not as sharp as assumed by Eq. 17. In particular, the intensity in pores that are only a few pixels thick is expected to be intermediate between $I_{0}$ and $I_{0}+\Delta I$. According to the threshold used to segment the tomogram, these pores can be arbitrarily left open or closed, which may have a significant impact on the binary tortuosity. For the gray-tone tortuosity, however, these pores are naturally considered to be half closed and half open. As the real pores are open, by definition, the point spread function is expected to bias the gray-tone tortuosity towards larger values. This is likely the reason for the differences between $\tau_{\mathrm{r}}$ and $\tau_{\mathrm{r}}^{\prime}$ for the sandstone (Table 1). No such effect is observed for the clay/plastic composite because the distance between neighboring clay lamellae is much larger than the pixel size.

\section{Relevance of a purely geometrical tortuosity}

The first question about tortuosity is whether it is mathematically well defined. For instance, if the relation between Euclidean and geodesic distances had not been linear, the question of the proportionality constant between $L_{\mathrm{G}}$ and $L_{\mathrm{E}}$ would have made no sense. It has been shown in "Nonuniqueness of the tortuosity" section that a material that is homogeneous on a relatively large scale is characterized by a joint distribution of the form of Eq. 12, which guarantees 
that tortuosity can be well defined. However, there is no unique definition of tortuosity. The two tortuosities $\tau$ and $\tau_{\mathrm{r}}$ measured in this article are related through

$$
\tau=\int_{1}^{\infty} x f(x) d x \text { and } \frac{1}{\tau_{\mathrm{r}}}=\int_{1}^{\infty} \frac{1}{x} f(x) d x
$$

and the two values coincide only for very peaked functions $f(x)$, which is the case for very simple porous structures as the one sketched in Figure 11. When using the simple concept of tortuosity, the complex morphological information contained in $f(x)$ is condensed into a single numerical factor.

The second question about tortuosity concerns the relevance of the proposed measures of tortuosity for the prediction of transport properties in porous materials. As discussed in the introduction, the tortuosity that is dealt with in this paper is purely geometrical. It is based on the length of the shortest path between two points in a pore network, irrespective of the possible pore constrictions. In the case of diffusion and of electrical conduction, the transport across a given section is proportional to the area of that section, so that bottleneck effects are expected to play an important role. This may explain why both $\tau$ and $\tau_{\mathrm{r}}$ underestimate the diffusional tortuosity of the FPS model at intermediate porosity (see full symbols in Figure 4).

The underestimation is expected to be more critical for fluid permeability; the no-slip boundary condition of the fluid on the pore wall results in the transport being proportional to the square of the section area. ${ }^{7}$ In this context, some authors propose to defined tortuosity as a weighted average using the volumetric flow along each streamline as a weighting factor, ${ }^{16}$ which may lead to significantly larger values that the approach presented here. For many materials, however, the tortuosity determined from electrical conductivity (which is expected to be the same as from diffusion) enables a quantitative prediction of fluid permeability. ${ }^{17}$

These considerations notwithstanding, the estimated values of the tortuosity (both $\tau$ and $\tau_{\mathrm{r}}$ ) correlate globally very well with the effective diffusion coefficients for the series of FPS models of various densities (Figure 2). Despite the simplicity of the model, FPS is known to be a realistic model of the morphology of consolidated media such as sandstones and sintered materials. ${ }^{14,18}$ Therefore, the estimation of tortuosity according to the methods presented in this article may well provide a useful link between microstructure and macroscopic transport for a large class of materials. In addition, the graytone tortuosity can also be used in the analysis of compounds where the phases have different, but all finite, permeabilities.

\section{Conclusions}

We have presented two different methods for measuring the tortuosity of porous solids from their tomographic reconstruction. The first method is based on the direct comparison of the geodesic and Euclidean distances calculated from any pore pixel to any limit of the tomogram. The second method is based on the geodesic reconstruction of the pore space of the tomogram, starting from any of its limits. The first method is restricted to binary tomograms; it therefore requires a preliminary segmentation step by which the user defines explicitly which pixels belong to the pore and solid phases of the material. The method based on geodesic reconstruction is more general; it can be applied to gray-tone tomograms without any segmentation step. In the case of a simple structure made up of nonconnected narrow pores, the two methods give the same result. For more complex and realistic structures, however, the two measures of the tortuosity are different.

The fact that tortuosity can be measured in different ways points to the fact that its mathematical definition is not unique. A complete description of the sinuosity of pore space is given by the joint statistical distribution of Euclidean and geodesic distances, which-under the general hypothesis of material's homogeneity - can be described with a single function as $f\left(L_{\mathrm{G}} / L_{\mathrm{E}}\right)$. When different methods are used to measure the tortuosity from the tomograms, the complex information contained in $f\left(L_{\mathrm{G}} / L_{\mathrm{E}}\right)$ is condensed differently into a single number, in a predictable way. Furthermore, when tortuosity is measured on gray-tone tomograms, the effect of low contrast and low resolution of the imaging technique can also be understood: a low contrast leads to an underestimation of the tortuosity; a low resolution leads to an overestimation.

The tortuosity dealt within the article is a geometrical tortuosity that is insensitive to the constriction effects that can play an important role in transport phenomena. For practical purposes, however, the tortuosities measured in this article compare very well with effective diffusion coefficients, in the case of realistic morphological models of a large class of porous materials. Therefore, not only can the methods proposed in this article help identifying the determinants of measured transport properties, but they may also prove useful for predicting transport properties from the tomographic reconstruction of the materials.

\section{Acknowledgments}

C.J.G. is a postdoctoral researcher of the Belgian Funds for Scientific Research (FRS-FNRS). This work was supported by the FRS-FNRS, the Région Wallonne-Direction Générale des Technologies de la Recherche et de l'Energie and the Interuniversity Attraction Poles Program-Belgian Science Policy P6/17. The sandstone microtomography data was generated on beamline X-2B at the National Synchrotron Light Source which is supported by ExxonMobil Research and Engineering Company. Use of the National Synchrotron Light Source, Brookhaven National Laboratory, was supported by the U.S. Department of Energy, Office of Science, Office of Basic Energy Sciences, under Contract No. DE-AC02$98 \mathrm{CH} 10886$.

\section{Literature Cited}

1. Weyland M, Midgley PA. Electron tomography, Mater Today. 2004; 7:32-40.

2. Kubis AJ, Shiflet GJ, Dunn DN, Hull R. Focused ion-beam tomography, Metall Mater Trans A. 2004;35:1935-1943.

3. Fredrich JT, Menéndez B, Wong T-F. Imaging the pore structure of geomaterials, Science. 1995;268:276-279.

4. Fredrich JT. 3D imaging of porous media using laser scanning confocal microscopy with application to microscale trasport processes, Phys Chem Earth A. 1999;24:551-561.

5. Möbus G, Inkson BJ. Nunoscale tomography in materials science, Mater Today. 2007;10:18-25.

6. Adler PM. Porous Media: Geometry and Transport. Boston: Butterworth-Heinemann, 1992.

7. Clennell MB. Tortuosity: a guide through the maze. In: Lovell MA, Harvey PK, editors. Developments in Petrophysics, Vol. 122. London: Geological Society Special Publication, 1997:299-344.

8. Satterfield CN. Mass Transfer in Heterogeneous Catalysis. Cambridge: MIT Press, 1970.

9. Kuebel C, Voigt A, Schoenmakers R, Otten M, Su D, Lee TC, Carlsson A, Bradley J. Microsc Microanal. 2005;11:378-400. 
10. Soille P. Morphological Image Analysis, Principles and Application. Berlin: Springer, 1999.

11. Dougherty ER, Lotufo RA. Hands-on Morphological Image Processing. Bellingham: SPIE Press, 2003.

12. Flannery BP, Deckman HW, Roberge WG, D'Amico KL. Three Dimensional X-ray microtomography, Science. 1987;237:1439-1444.

13. Dunsmuir JH, Ferguson SR, D'Amico KL, Stokes JP. X-ray microtomography: a new tool for the characterization of porous media, paper SPE 22860. Proceedings of the 1991 SPE Annual Technical Conference and Exhibition, Dallas, October 6-9, 1991.

14. Serra J. Image Analysis and Mathematical Morphology, Vol. 1. London: Academic Press, 1982.

15. Kim IC, Torquato S. Diffusion of finite-size Brownian particles in porous media. J Chem Phys. 1992;96:1498-1503.

16. Knackstedt MA, Zhang X. Direct evaluation of length scales and structural parameters associated with flow in porous media, Phys Rev E. 1994;50:2134-2138.

17. Katz AJ, Thomson AH. Quantitative predication of permeability in porous rock, Phys Rev B. 1986;34:8179-8181.

18. Torquato S. Random Heterogeneous Materials: Microstructure and Macroscopic Properties. New York: Springer, 2002.

\section{Appendix A: Arguments for the Exponent of Tortuosity in Eq. 2}

Equation 2 of the main text, which relates the effective diffusivity in a porous solid to the tortuosity, is different from that found in several textbooks (e.g., Ref. 8) as it contains the square of the tortuosity. In most cases, this imprecision is not detrimental because tortuosity is commonly treated as a numerical factor, and no attempt is done to give it a precise geometrical meaning. In this appendix, we show that Eq. 2 of the main text is correct if the tortuosity is to be interpreted as the ratio of the diffusive path lengths to the straight length across the solid. We give two different arguments. The first is based on stationary diffusion in a particular pore structure; the second is not limited to any particular pore structure and it is based on transient diffusion.

Following Satterfied, ${ }^{8}$ let us imagine a vertical slab of thickness $L$, cut out of a simplified porous solid made up of cylindrical pores of cross section $\Omega$, all having an angle $\theta$ with the direction perpendicular to the slab. The length of each pore is therefore $L^{\prime}=L / \cos (\theta)$. Let there be $n$ such pores per unit surface of the slab. The porosity is easily found to be

$$
\phi=n \Omega \frac{L^{\prime}}{L}
$$

If a concentration difference $\Delta c$ in any molecule is set across the slab, each pore will individually contribute to transport a molar flow of

$$
N=\Omega D_{\mathrm{m}} \frac{\Delta c}{L^{\prime}}
$$

where $D_{\mathrm{m}}$ is the molecular diffusion coefficient. The total macroscopic molar flow is obtained by summing over all pores, which introduces simply a factor $n$ in Eq. A2. Combining Eqs. A1 and A2 leads to

$$
\sum N=\phi \frac{D_{\mathrm{m}}}{\left(L^{\prime} / L\right)^{2}} \frac{\Delta c}{L}
$$

which enables us to identify $\phi D_{\mathrm{m}}\left(L / L^{\prime}\right)^{2}$ with the effective diffusion coefficient. The ratio $L^{\prime} / L$ is nothing but the tortuosity; it appears here naturally with exponent 2 , in agreement with Eq. 2 of the main text.

The second argument is more general. It is based on the fact that the time needed for a molecule to diffuse over a given distance scales with the square of that distance (e.g., Bird et al., Transport Phenomena, New York, Wiley, 1960). Taking a slab of porous material of thickness $L$, and increasing suddenly the concentration of any molecule on one side, the concentration on the other side will start increasing after a time

$$
t \approx\left(L^{\prime}\right)^{2} / D_{\mathrm{m}}
$$

where $L^{\prime}$ is the actual distance the molecules have to diffuse along, and $D_{\mathrm{m}}$ is the diffusion coefficient inside the pores. If we wish to describe the same experiment with an effective diffusion coefficient $D_{\mathrm{e}}$ through the material ignoring the tortuous path of the molecule, we would write

$$
t \approx L^{2} / D_{\mathrm{e}}
$$

where $L$ is the thickness of the slab. As the time $t$ needed for the molecules to make their way through the slab does not depend on the modeling methodology, the comparison of Eqs. A4 and A5, lead to

$$
\frac{D_{\mathrm{e}}}{D_{\mathrm{m}}} \approx\left(\frac{L}{L^{\prime}}\right)^{2}
$$

which shows that the effective diffusion coefficient is proportional to the square of the tortuosity. The reason why the porosity $\phi$ does not appear in Eq. A6 is that it is based only on the time needed for a molecule to travel across the material and not about how many molecules are involved.

\section{Appendix B: Geodesic Distance and Geodesic Reconstruction}

\section{Geodesic distance}

The geodesic distance between two points belonging to a given set is the length of the shortest path between these two points that is included in the set. ${ }^{9,11}$ This is illustrated in Figure B1 in the case of a model image obtained by randomly dropping disks in an image (Figure B1, a), which is the 2D equivalent of the FPS model of the main text (Figure 3).

The Euclidean distance between any pixel in the white phase and the left limit of the image is measured as the crow flies, i.e. possibly across the black phase (Figure B1, b). It does not depend on the geometry of the black and white phases. The Geodesic distance between any point of the white phase and the left limit of the image is the length of the shortest path from the left limit of the image to that point within the white phase. It is the length of the shortest path that avoids the black phase (Figure B1, c).

The geodesic distance between two points is therefore generally larger than the Euclidean distance; both distances are equal only if there exists a straight path between the two points, within the white phase. 

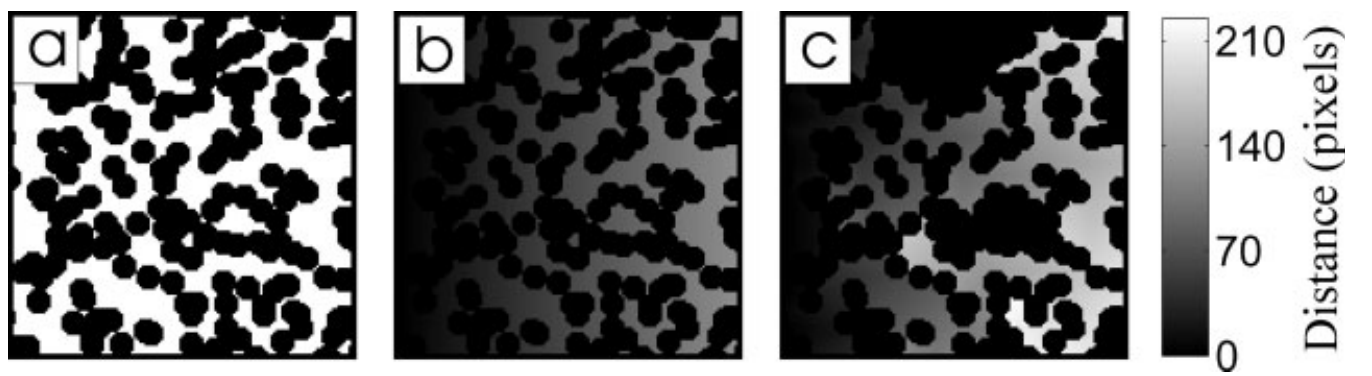

Figure B1. Binary image (a), and the Euclidean (b) and geodesic (c) distances from each white pixel to the leftmost limit of the image.

The Euclidean distance is the shortest distance across the white and black zones, and the geodesic distance is the shortest distance within the white region.

\section{Geodesic reconstruction}

The principle of geodesic reconstruction is illustrated in Figure B2, in the case of a $4 \times 4$ pixels gray-tone image (Figure B2, i). The intensity of an image is synonymous to its luminosity: bright pixels correspond to large intensities and dark pixels to low intensities. For images coded on 8 bits, the intensity ranges from 0 (black) to 255 (white). There are 4 different intensities in image $\mathrm{i}$ of Figure B2: 10 (dark gray), 50 (gray), 150 (bright gray), and 255 (white).

Every individual step of the geodesic reconstruction, from image $r_{0}$ to $r_{1}$, from $r_{1}$ to $r_{2}$, and so forth, is a geodesic dilation; it consists in the subsequent dilation of the image by a given structuring element SE (symbolized by $\oplus \mathrm{SE}$ ), and its intersection with the original image (symbolized by $\cap$ ), as explained hereafter. The starting image for the geodesic reconstruction (Figure B2, $\mathrm{r}_{0}$ ) is the leftmost column of image i.

The dilation of a gray-tone image by a structuring element $\mathrm{SE}$ is obtained in the following way. The structuring element $\mathrm{SE}$ is moved in the image; for every possible position of SE, the intensity of the pixel in the center of SE is replaced by the largest intensity of the image all over SE. For an image digitized on a square grid of pixels, a convenient structuring element is a cross containing 5 pixels, as in Figure B2. In that figure, $d_{1}$ is obtained by dilating $r_{0}$ by SE, $d_{2}$ is obtained by dilating $r_{1}$ by $S E$, and so forth.

Intersecting two gray-tone images consists in keeping, pixel by pixel, the lowest value of both images, i.e., the darkest intensity. In Figure B2, $r_{1}$ is the intersection of $d_{1}$ and $i, r_{2}$ is the intersection of $d_{2}$ and $i$, and so forth. In the particular case of two binary images, with only possible values 0 and 1 , this coincides with the classical definition of the intersection of two sets, as the intersection takes the value 1 if and only if the two intersected images have the value 1 at that pixel.

The geometrical interpretation of the geodesic reconstruction is clear when looking at Figure B2, from $r_{0}$ to $r_{4}$. The bright zone is propagated and when a darker zone is met, only the darkest intensity encountered can be propagated further. For instance, the white pixel in the upper right corner is never recovered in the reconstruction because it is surrounded by dark pixels.

In the particular case of a binary image, the geodesic reconstruction can be used to measure a geodesic distance. This is illustrated in the first row of Figure B3, in which the same binary image as in Figure B1 is reconstructed. The

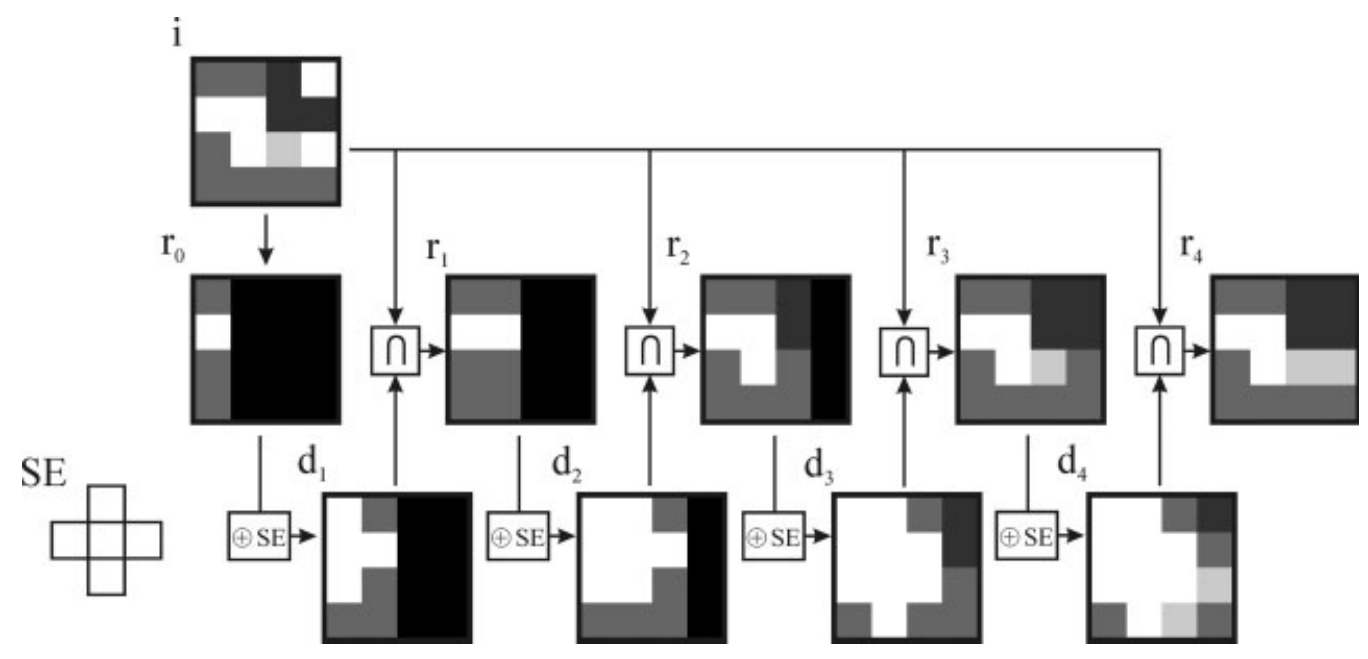

Figure B2. Principle of gray-tone geodesic reconstruction: each geodesic dilation consists in the dilation of the image by a structuring element SE, followed by the intersection of the dilated image d with the original image $\mathrm{i}$. 

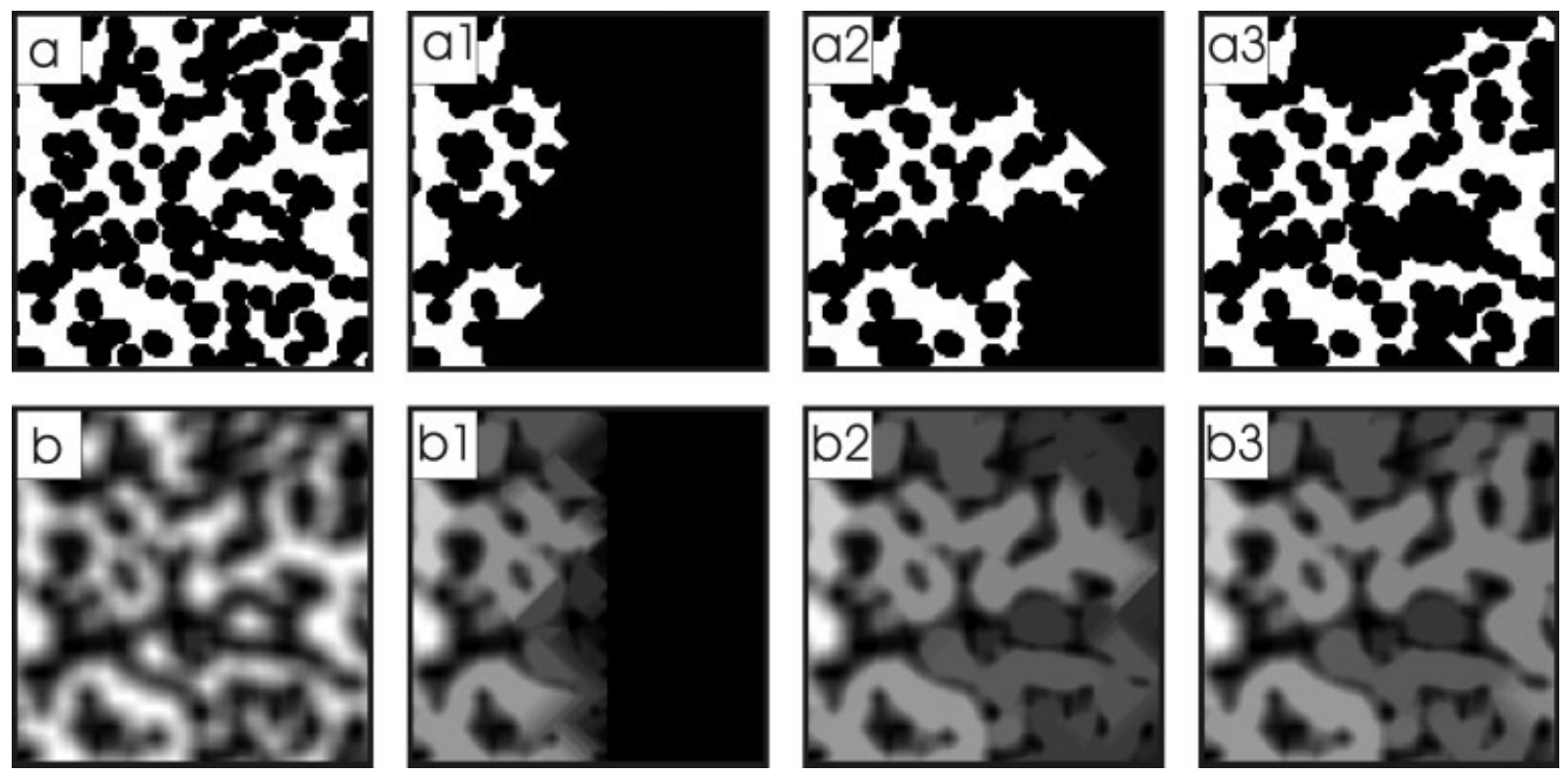

Figure B3. Progressive geodesic reconstruction of a binary (a) and of a gray-tone (b) images. The images in a1 (b1), a2 (b2), and a3 (b3) were obtained after 70, 140, and 210 geodesic dilations.

geodesic distance from the left limit of the image to any white pixel can be defined as the number of geodesic dilations needed for the reconstructed area to reach that pixel. It is readily checked that the white pixels in Figure B3 a1 (a2, a3) coincide with the pixels of Figure B1 c in which the distance is lower than $70(140,210)$.

Unlike the geodesic distance that is defined for binary images only, the geodesic reconstruction can be applied to gray-tone images as well (second row of Figure B3). The gray-tone image in Figure B3 b was obtained from the binary image in Figure A3 a by replacing every pixel by the average intensity over a $9 \times 9$ pixels neighborhood. The geodesic reconstruction of the latter image from its leftmost limit is illustrated in Figure B3 b1 to b3.

Manuscript received Sept. 15, 2008, and revision received Dec. 10, 2008. 